\title{
Oscillations of Force in the Standing Legs of a Walking Insect (Carausius morosus) *
}

\author{
H. Cruse and G. Saxler \\ Fachbereich Biologie, Universität Kaiserslautern, FRG
}

\begin{abstract}
In the experiments presented here adult stick insects (Carausius morosus) walk on a treadwheel with various legs standing on platforms fixed relative to the body of the insect. These standing legs produce large forces directed towards the rear which are modulated in the rhythm of the walking legs. Neighbouring legs which both stand on a platform often oscillate "in phase". Possible reasons for the occurrence of the force oscillations are discussed.
\end{abstract}

\section{Introduction}

The purpose of this investigation is to obtain information upon the nature of those six neuromuscular subsystems which control the movement of the six legs of an insect and upon the nature of the connections between these subsystems which produce a coordinated pattern in the walking animal. However, it is very difficult to draw necessary conclusions about this system for the following reasons. Firstly one has a system with six output channels (the force or the position of each leg) and an unknown number of input channels which can be quantitatively manipulated either not at all (central commands) or only with difficulty (influences from sense organs). Furthermore these inputs are usually dependent upon the values of the output channels (as measured by sense organs on the legs). Secondly one does not know whether the connections between the six subsystems are "hard wired" in the sense that they work in the same way in all the experimental situations investigated or whether some of them can be changed (e.g. switched off or altered) depending upon the experimental situation. Thirdly one does not know the structure of the individual subsystems.

* Supported by DFG (Cr $58 / 1)$
One possible approach to this problem is to build up hypothetical models as Wendler (1968, 1978), Graham $(1972,1977)$, Cruse (1979). Such a quantitative hypothesis can then be tested by comparing the calculated results with the largest possible number of experimental results. Particularly interesting for such tests are experimental situations which do not occur in the normal life of the animal but leave the whole system intact.

For a walking animal a situation of this kind was first discovered by Wendler (1964) and later used by Bässler (1967). A stick insect Carausius morosus was fixed dorsally on a holder and was allowed to walk on a treadwheel. Then one leg was placed on a platform fixed relative to the body of the insect beside the treadwheel. If the position of the platform is far enough forward, this leg remains standing on the platform while the other legs walk and turn the treadwheel. The coordination pattern of the walking legs may be changed by such an experiment (Wendler, 1964). Wendler (1964) and Bässler (1967) found that the standing leg applies a force to its platform. When using sensitive electronic force transducers our own experiment showed that the force of the standing leg was modulated in the rhythm of the walking legs. This method was also used by Bässler (1979) with Extatosoma tiaratum which showed a similar behaviour. In this paper, the phase relations of both walking legs and the force oscillations of the standing legs are investigated by varying the number and the arrangement of the walking and standing legs.

\section{Methods}

Adult female stick insects (Carausius morosus) are restrained by the dorsal thorax in a holder with the longitudinal axis of the body horizontal. The animal can walk on a treadwheel made from styrofoam with a diameter of $38.5 \mathrm{~cm}$ and a breadth of $9 \mathrm{~mm}$. The moment of inertia is $720 \mathrm{gcm}^{2}$. The friction of $0.8 \mathrm{mN}$ is measured as 


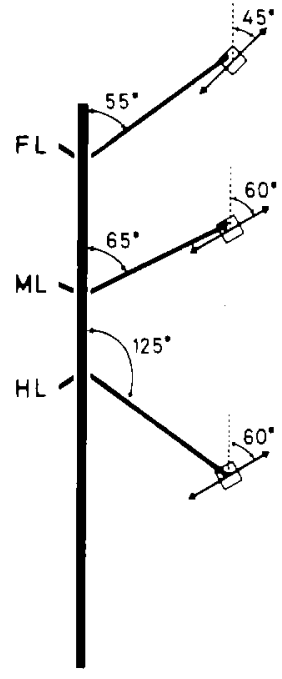

Fig. 1. The position of the legs and the direction of forces measured when the legs stand on force transducers. FL: frontieg, ML: middleleg, HL: hindleg
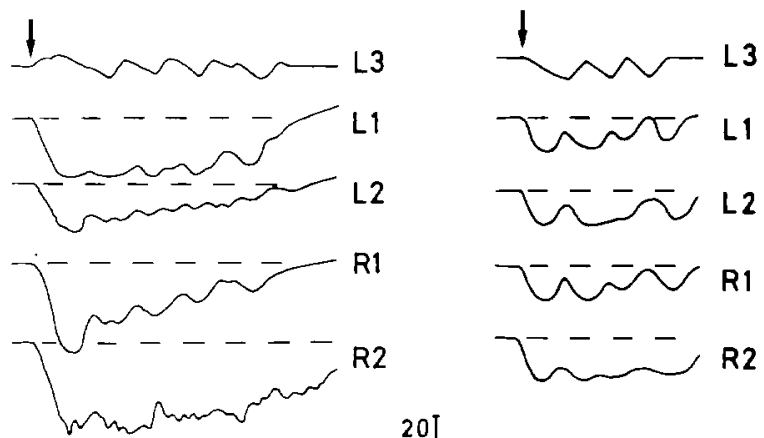

$$
1
$$

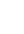
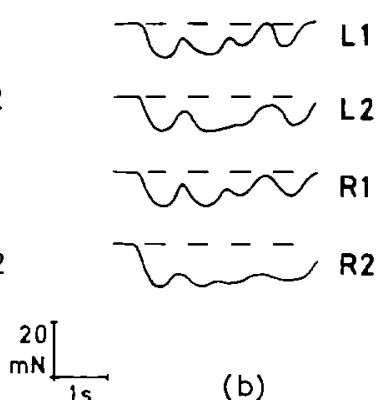

(b)

Fig. 2a and b. Experimental situation L3R3. The upper trace shows the movement of the walking leg L3 (retraction downward, protraction upward). The other traces show the forces developed by the legs L1, L2, R1, and R2. The zero force levels are shown by interrupted lines. The forces act backwards with exact direction of measured component shown in Fig. 1. The movements of the walking legs not shown are investigated in separate experiments

that force applied to the outer margin of the treadwheel that is necessary to move the wheel continuously. In later control experiments with a treadwheel of a moment of inertia of $400 \mathrm{gcm}^{2}$ and a friction of $<0.1 \mathrm{mN}$ similar force oscillations can be seen. As the holder is rigid the legs of the animal do not have to support the weight of the body.

In the ten different experimental situations investigated here certain of the six legs were placed on individual platforms, the position of each was fixed relative to the animal's body. Each platform was mounted on a force transducer (strain gauge). The components of the forces which were measured in these experiments are shown in Fig. 1. These directions were chosen such that maximum amplitudes of the oscillating forces were obtained. The amplitude of movement of the platform for the maximum value of the force lay between $0.2 \mathrm{~mm}$ and $2 \mathrm{~mm}$ depending upon the type of force transducer and had a maximum value of $0.2 \mathrm{~mm}$ during the oscillation of the forces. No measurable forces were transferred across the body from one platform to another.

As the form of the force oscillations sometimes is not symmetrical the phase position of the minimum of the force as well as the phase position of the maximum of the force relative to the reference leg was recorded. As however, the final phase plots showed a corresponding shape only the distributions of the force maximum values are shown in the tables. When the force oscillations of two standing legs are compared with each other then it is always the maximum that is used as reference point To combine the force oscillations of the standing legs with the movement of the walking legs the movement of one walking leg, the reference leg, is recorded by using a position diode [UDT, Typ PIN SC/10, for details see v. Helversen and Elsner (1977), Pflüger (1977) and Saxler (1976)]. Thereby the position of the femur of the reference leg was measured.

Concerning the coordination of two legs they are said to be either "in phase" or to "alternate" (for quantitative definition see Cruse and Saxler, 1980). Sometimes also bimodal phase distributions seem to occur.

To describe the different experimental situations the following abbreviations for the six legs are used: L1 left frontleg, L2 left middleleg, L 3 left hindleg, R1 right frontleg, R 2 right middleleg and $\mathrm{R} 3$ right hindleg. The experimental situations themselves are named by the underlined abbreviation of those legs which walk free in this situation. For example in the situation L1L2R1R2 both front- and middlelegs walk, whereas each hindleg stands on the platform of a force transducer. The following ten experimental situations are investigated: L1L2R1R2 L2L3R2R3, L1L3R1R3, L1R1, L2R2 L3R3, L1, L1L2, L1L2L3, and L2

\section{Results}

Two examples of the force measurements are given in Fig. 2 (experimental situation L3R3). When the animal starts to walk (Fig. 2, arrow) the forces of all legs increase. During the walk of L3 and R3 the standing legs show a modulated force output. To obtain an estimation of the change of the $d c$-value of the force for different walking periods the $d c$-value of the forces is estimated by eye. The following values give the mean values of $n$ values measured by this way: frontleg 18.4 (S.D. \pm 2.0$) \mathrm{mN}(n=52)$; middleleg 20.5 (S.D. \pm 2.2$) \mathrm{mN}$ $(n=41)$; hindleg 6.3 (S.D. \pm 2.0$) \mathrm{mN}(n=75)$. These values are much higher than forces applied by the legs of free walking animals which are in the order of magnitude of about $1 \mathrm{mN}$ (Cruse, 1976).

The modulation of the forces in all the experimental situations most often occurs with the same frequency as the walking legs and is coordinated with them. In some cases it is double the frequency and occasionally no coordination is observed between walking movement of a leg and the force in a standing leg. This contributes towards the large standard deviation of most of the phase distributions.

In other cases (Fig. 3, experimental situation L2L3R2R3) the coordination is so strong between the standing legs that over a large time interval every small change in the force values is nearly identical for both legs. However, this "rigid coupling" may also momentarily disappear so that some other coordination be- 
tween the two legs can be seen. Other examples of "in phase" coordination between standing legs are shown in Fig. $2 \mathrm{~b}$ and in Fig. 4 (experimental situation L1L3R1R3). In Fig. 5 (experimental situation L1L2R1R2) both standing hindlegs show an alternating coordination.

In the following some additional qualitative results are stated while the detailed quantitative results will be shown together with the results concerning the coordination of the other walking legs (Cruse and Saxler, 1980). The force oscillations of standing legs most often are "in phase". If in the phase distribution two modes occurred then this is true for one of these modes. This is the case for all standing legs in the following experimental situations: L2L3R2R3, L1R1, L1, $\underline{\text { L1L2L3 }}$, and L1L2. Only in the last experimental situation mentioned between the legs $\mathrm{R} 2$ and $\mathrm{R} 3$ is a bimodal distribution obtained with both modes of $\mathrm{R} 3$ alternating. In the following experimental situations only some pairs of legs are obviously in phase, $\underline{\mathrm{L} 2 \mathrm{R} 2}$ : L1-R1, R1-R3, L2: L1-R1, R1-R2, the other pairs show no detectable coordination. Only the following standing legs are alternating with a phase shift of about $180^{\circ}$ : L1L2R1R2: L3-R3, L2: L3-R3. As will be shown in the following paper (Cruse and Saxler, 1980) neighbouring (ipsilateral and contralateral) walking legs are either alternating or there is no detectable coordination. No constant "in phase" coordination between neighbouring walking legs was observed. Only in one situation (L1L2R1R2) does the coordination of all legs agree with that of a free walking animal (see also Cruse and Saxler, 1980, Fig. 1). In all the other experimental situations the coordination is different.

The experimental results show that the connections between two subsystems seem to be variable. If two legs in one of the experimental situations are strongly "in phase" this may not be the case in another experimental situation. But also within the same experimental situation some variability exists as described above.

\section{Discussion}

Where do the movements of the walking legs and the force oscillations in standing legs come from? Several different possibilities are discussed. It may be that to produce the coordination pattern of walking legs there exists only one central rhythm generator which gives commands to the different legs to start pro- or retraction, and is not influenced by any sensory feedback. Then the change of force in the standing leg could be the result of such a command. From the experimental results such a hypothesis cannot be the only mechanism as in all the experimental situations

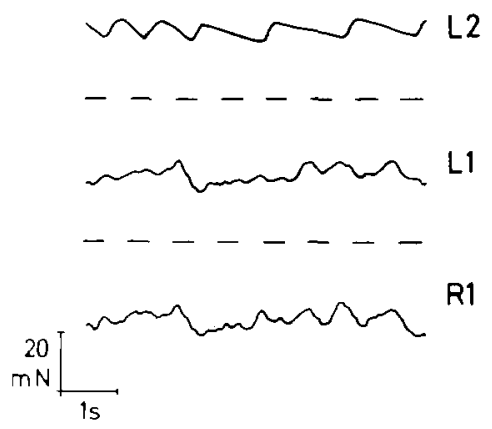

Fig. 3. Experimental situation $L 2 L 3 R 2 R 3$. The upper trace shows the movement of the walking leg L2, the other traces show the forces developed by the standing legs L1 and R1. See also text of Fig. 2

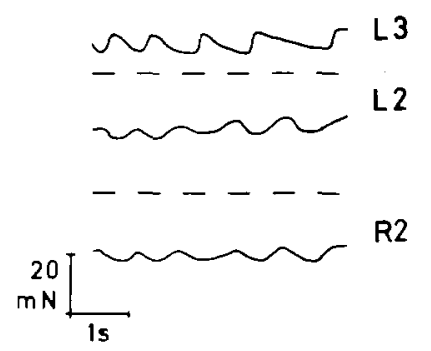

Fig. 4. Experimental situation L1L3R1R3. The upper trace shows the movement of the walking leg L3. The other traces show the forces developed by the standing legs L2 and R2. See also text of Fig. 2

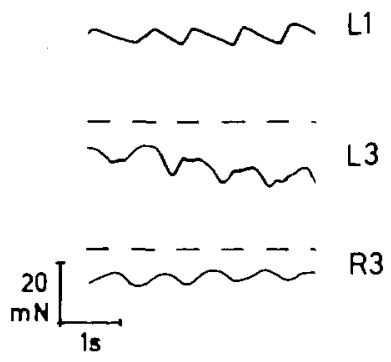

Fig. 5. Experimental situation L1L2R1R2. The upper trace shows the movement of the walking leg L1. The other traces show the forces developed by the standing legs L3 and R3. See also text of Fig. 2

one would expect the same type of coordination except for those changes which depend upon different walking speeds. Therefore one can say that the force development and the movement of the legs is not controlled by only one central program. It is much more probable that each leg is controlled by single neuro-muscular subsystems and the connections between them are variable depending on the sensory feedback. This is supported by results of other authors which show that at least different groups of legs can work independently from one another. Wendler (1964) showed relative coordination between front- and hindlegs of stick insects when walking on a treadwheel with the middlelegs amputated. Graham showed that the legs on 
each side of the body can step at a different frequency when turning (1972) and when walking on a treadwheel with two independent wheels one for the legs of each side of the body (Graham, 1980).

What can be said concerning the subsystem itself, which controls the movement of an individual leg? An often discussed alternative is whether the cyclic movements of a leg are controlled by a pure "central oscillator" with sensory influences which perform only modulations or whether they are produced by a chain reflex which means that commands from the periphery are necessary for the continuation of a cyclic movement, this may be called a "peripheral oscillator". As there exist experimental results which strongly support the central oscillator hypothesis (Pearson and Iles, 1970 ) and others which strongly support the concept of a peripheral oscillator (e.g. Ten Cate, 1936, for discussion see Wilson, 1966) these alternative ideas seem to be misleading. Several results of Bässler (1977) and Graham and Bässler (1980) show that the cyclic movement can be interrupted at different positions when sense organs may be signalling that a definite part of the movement cycle has not yet been finished. This is also indicated by the experiment presented here. The standing leg does not produce a protraction movement if it has not reached a sufficiently posterior position, although protraction commands from other subsystems are to be expected. We also observed a corresponding effect during the protraction movement. When during a walk on the treadwheel the protraction movement of a leg is stopped by holding a stick vertically in front of the femur, then the leg was never observed to finish the protraction by depressing the leg and to start retraction movement but continuously tries to protract. The leg sometimes moves a little backwards and tries again to protract with a higher position of the femur possibly to pass over the obstacle. Although the other legs go on walking the behaviour of this leg continues until the leg by chance finds tarsal contact. This indicates that sensory feedback can also prevent the leg from changing over from protraction to retraction. On the other hand after ablation of all the sense organs which were investigated by Bässler (1977) the individual legs showed none of the particular changes described by Bässler but showed a more or less normal coordination pattern (Cruse and Pflüger, in prep.). All these results together show that the subsystem does not work like a chain reflex which must have sensory feedback to initiate the following reflex movement but that at least in these special cases sensory influence prevents the transition from one part of the cycle to another (for discussion see also Cruse, 1980).

According to these results a subsystem might be constructed in two different ways. The subsystem could consist of $(a)$ an oscillator which itself can be influenced (e.g. stopped) at different cycle positions. As the second possibility $(b)$ the oscillator itself is not influenced by the sensory feedback but goes on running although the leg stands. This means that the subsystem could be regarded as consisting of two parts a "central" and a "peripheral" part which are connected in the normal walking leg but could be uncoupled by sensory influence. Then the sensory influence stops the peripheral part but not the central one which goes on performing oscillations.

Although a clear cut decision between these two hypotheses at the moment is impossible, the following experiment supports the hypothesis that the subsystems correspond to the type $(a)$. When an animal walks on the treadwheel and this is suddenly stopped by hand, the force oscillations of the standing legs do not stop immediately but show further activity for several cycles. This can be seen most readily when one or more legs show further protraction movements after the wheel has stopped. If no leg is moved after the wheel stops, then the forces of all legs increase during the first $0.5 \mathrm{~s}$ and within few seconds fall with no detectable oscillations to a small constant value. If a central oscillator of a standing leg were the cause of movements in another leg then at least occasionally one would expect to find force oscillations without movements in other legs. This has not so far been observed in stick insects. On the contrary it has sometimes been noticed that walking legs moved while no force oscillation in standing legs were detectable. The results suggests that the fundamental cause of the force oscillations of a standing leg must be found in the subsystem of a "moving" leg.

Where could the force oscillations found in standing legs come from? In the first case (a) the oscillator of a standing leg is stopped. Therefore the force oscillations could only be produced by influences from other subsystems of either walking or standing legs. The latter however according to this hypothesis do not produce their own rhythm but can only transfer the input values obtained from other subsystems. Therefore basically the oscillations are produced by the subsystems of walking legs. This means that only subsystems of walking legs can work as pacemakers. When subsystems of type $(b)$ are assumed to exist then the force oscillations could also be produced by the oscillations of the central part of the subsystem of the standing leg itself. Then also the subsystem of a standing leg could act as pacemaker. As there were no force oscillations which were not coordinated with one of the other legs the oscillations of the central part may be produced by the other subsystems except for those which might themselves be a pacemaker. This means that in both cases the oscillations of most of the legs 
are the result of influences from other subsystems except for those which function as the pacemakers.

The most obvious result of the experiments reported here is the occurrence of the force oscillations in standing legs and that these oscillations are most often "in phase". It was stated earlier that this "in phase" coordination cannot be seen in a "normal" walking animal. It should however be mentioned that under special conditions similar effects can be observed which possibly could be related with this phenomenon. When stick insects walk vertically upwards or when they walk pulling against a weight or on a treadwheel with high friction, legs of the same segment can protract at the same time. Also the effect mentioned earlier that the force of all legs standing on force transducers is increased when the treadwheel is stopped by hand may be based upon the same mechanism.

Acknowledgements. We want to thank to Prof. Dr. U. Bässler and Dr. D. Graham for many helpful discussions.

\section{References}

Bässler, U.: Zur Regelung der Stellung des Femur-Tibia-Gelenkes bei der Stabheuschrecke Carausius morosus in der Ruhe und im Lauf. Kybernetik 4, 18-26 (1967)

Bässler, U.: Sensory control of leg movement in the stick insect Carausius morosus. Biol. Cybernetics 25, 61-72 (1977)

Bässler, U. : Interaction of peripheral and central mechanisms during walking in the 1st instar Extatosoma tiaratum. Physiol. Entomol. 4, 193-199 (1979)

Cruse, $\mathrm{H}$.: The function of the legs in the free walking stick insect, Carausius morosus. J. Comp. Physiol 112, 235-262 (1976)

Cruse, H.: A new model describing the coordination pattern of the legs of a walking stick insect. Biol. Cybernetics 32, 107-113 (1979)

Cruse, H.: A quantitative model of walking incorporating central and peripheral influences. I. The control of the individual leg. Biol. Cybernetics (in press) (1980)

Cruse, H., Pflïger, H.J. : Is the position of the femur-tibia-joint under feedback control in the walking insect? (In preparation)

Cruse, H., Saxler, G.: The coordination of force oscillations and of leg movement in a walking insect (Carausius morosus). Biol. Cybernetics 36, 165-171 (1980)
Graham, D.: A behavioural analysis of the temporal organization of walking movements in the 1st instar and adult stick insect (Carausius morosus). J. Comp. Physiol. 81, 23-52 (1972)

Graham, D.: A model for the control of coordinated leg movements in free walking insects. Biol. Cybernetics 26, 187-198 (1977)

Graham, D.: Walking dynamics of the stick insect using a counterbalanced low inertia, independent double tread wheel. (1980)

Graham, D., Bässler, U.: Effects of afference sign reversal on the motor activity in walking stick insects (Carausius morosus). (1980)

Helversen, O. v., Elsner, N.: The stridulatory movements of acridid grasshoppers recorded with an opto-electronic device. J. Comp. Physiol. 122, 53-64 (1977)

Pearson, K.G.: Des, J.F.: Discharge patterns of coxal levator and depressor motoneurones of the cockroach, Perlplaneta americana. J. Exp. Biol. 52, 139-165 (1970)

Pfluiger, H.-J.: The control of rocking movements of the phasmid Carausius morosus Br. J. Comp. Physiol. 120, 181-202 (1977)

Saxler, G.: Die Beeinflussung der Beinkoordination durch Veränderung der sensorischen Rückmeldungen bei der Stabheuschrecke Carausius morosus. Examensarbeit, Universität Kaiserslautern 1976

Ten Cate, J. : Beiträge zur Innervation der Lokomotionsbewegung der Heuschrecke Locusta viridissima. Arch. Neerl. Physiol. 21, 562-566 (1936)

Wendler, G.: Laufen und Stehen der Stabheuschrecke Carausius morosus: Sinnesborstenfelder in den Beingelenken als Glieder von Regelkreisen. Z. Vgl. Physiol. 48, 198-250 (1964)

Wendler, G.: Ein Analogmodell der Beinbewegungen eines laufenden Insekts. In: Kybernetik 1968. Marko, H., Färber, G. (eds.). München, Wien: Oldenbourg 1968

Wendler, G.: Erzeugung und Kontrolle koordinierter Bewegungen bei Tieren. In: Kybernetik 1977. Hauske, G., Butenandt, E. (eds.). München, Wien : Oldenbourg 1978

Wilson, D.M.: Insect walking. Ann. Rev. Entomol. 11, 103-122 (1966)

Received: November 2, 1979

Prof. Dr. Holk Cruse

Gisela Saxler

Fachbereich Biologie der Universität

Postfach 3049

D-6750 Kaiserslautern

Federal Republic of Germany 